\title{
Linear-range Extension for Linear Variable Differential Transformer Using Binomial Series
}

\author{
Wandee Petchmaneelumka, Kanoknuch Songsuwankit," \\ Jakkapun Tongcharoen, and Vanchai Riewruja \\ Department of Instrumentation and Control Engineering, Faculty of Engineering, \\ King Mongkut's Institute of Technology Ladkrabang, Bangkok, 10520, Thailand \\ (Received April 11, 2019; accepted December 23, 2019)
}

Keywords: linear variable differential transformer, inductive transducer, linear range extension, binomial series, operational amplifier, analog multiplier

The linear-range extension technique for a linear variable differential transformer (LVDT) is described in this paper. Generally, the LVDT has a narrow linear operating range caused by its nonlinear transfer characteristic. To extend the linear operating range, the nonlinear behavior of the LVDT must be adjusted. In this paper, the circuit building block providing the LVDT inverse transfer characteristic using binomial series approximation is proposed for linearizing the nonlinear behavior of the LVDT. The third-order inverse transfer characteristic of the LVDT is synthesized from analog multipliers and a difference amplifier comprising an operational amplifier (opamp). All active devices used in this study are commercially available. Therefore, the attraction of the proposed technique is in the simple configuration and low cost, making it suitable for an embedded measurement system. The performance of the proposed technique is discussed in detail. Simulation and experimental results confirming the performance are also included. As a result, the linear range of the commercial LVDT used in this study can be extended more than $500 \%$. The full scale error of the measured value is about $0.23 \%$ over the entire operating range.

\section{Introduction}

An inductive transducer named the linear variable differential transformer (LVDT) provides the advantages of high resolution and durability. ${ }^{(1-3)}$ The LVDT is used in applications to measure displacement, which can be observed in the engineering, industrial, automotive, military, robotic, scientific, and medical equipment fields. ${ }^{(1-11)}$ The operation of the LVDT is based on the same principle as the transformer, which comprises a primary winding and two secondary windings with a moving core. ${ }^{(12,13)}$ The output signal of the LVDT is the difference signal between two secondary winding signals. Therefore, the LVDT signal is directly proportional to the position of the moving core that can be used to measure the displacement with high resolution. When the excitation signal is applied to the primary

*Corresponding author: e-mail: kanoknuch.so@kmitl.ac.th

https://doi.org/10.18494/SAM.2020.2407 
winding of the LVDT, the secondary windings generate the LVDT output signal in the form of an amplitude modulation with suppressed carrier (AMSC). ${ }^{(13)}$ Thus, the displacement signal can be extracted from the LVDT output signal using a synchronous demodulator based on a peak amplitude finder or an analog multiplier. ${ }^{(13-17)}$ The transfer characteristic of the LVDT provides a nonlinear behavior for the moving core that varies in the maximum stroke range, which can be expressed as a third-order function of the core position. ${ }^{(2,12,18)}$ Note that the linear operating range of the LVDT exists only in the narrow range close to the zero crossing. The large linear operating range of the LVDT with a compact structure is very useful for embedded measurement and instrumentation systems. However, the large linear operating range of the LVDT requires a large structure that is unsuitable for the embedded systems. In the past, the linear operating range of the LVDT was extended by the fractional order technique. ${ }^{(19)}$ Unfortunately, this technique is suited to a special design of the LVDT that causes a complex and large structure. The technique based on an artificial neural network (ANN) has been introduced in the literature. ${ }^{(20,21)}$ This technique can extend the linear operating range of the commercial LVDT using an adaptive inverse model. The use of the ANN technique requires a high-speed signal processor to calculate the adaptive inverse model. This is inconvenient for the embedded measurement system and uneconomical. Moreover, the ANN technique provides a large response time to obtain the linear displacement signal.

In this paper, the circuit building block providing the inverse transfer characteristic of the LVDT is proposed. The binomial series is used to estimate the inverse transfer characteristic of the LVDT. The LVDT output signal is demodulated to the displacement signal by the technique reported previously. ${ }^{(13)}$ The displacement signal is compensated to the linear displacement signal by the proposed scheme. Note that the proposed scheme can be implemented using only commercially available devices. The performance of the proposed technique is analyzed and discussed in detail. Experimental results show that the maximum percentage error of about $0.5 \%$ in the maximum stroke range is observed. The linear operating range of the commercial LVDT can be extended more than $500 \%$ by using the proposed technique. It is clearly seen that the proposed technique exhibits the advantages of fast response, high accuracy, simple configuration, and low cost.

\section{Basic Principle of LVDT}

The LVDT principle is similar to that of a transformer that comprises a primary winding $P$, two identical secondary windings, $S_{1}$ and $S_{2}$, and a moving core, as shown in Fig. 1(a). Two secondary windings are connected in opposed directions to generate the displacement signal. The amplitude of the displacement signal versus the core position is shown in Fig. 1(b). The structure of the LVDT is shown in Fig. 1(c), where the lengths of the primary winding and secondary windings are defined as $p$ and $w$, respectively, with the radius $d_{O}$. The number of turns in the primary winding and secondary windings are equal to $n_{p}$ and $n_{s}$, respectively. The dimensions of the LVDT core are obtained as the radius $d_{C}$ and length $L_{C}$. The insulators between the primary winding and the secondary windings have a thickness of $g$. The sinusoidal signal $v_{e x}=V_{e x} \sin \left(\omega_{e x} t\right)$ is applied as the excitation signal for the primary winding, where $V_{e x}$ 


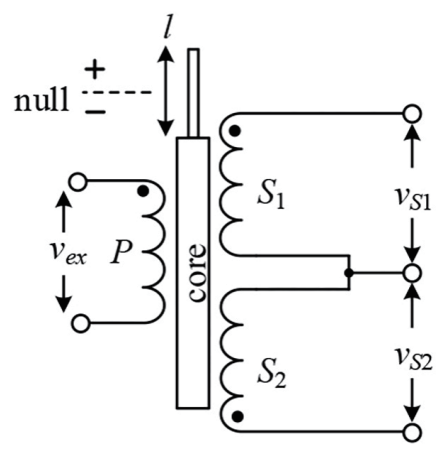

(a)

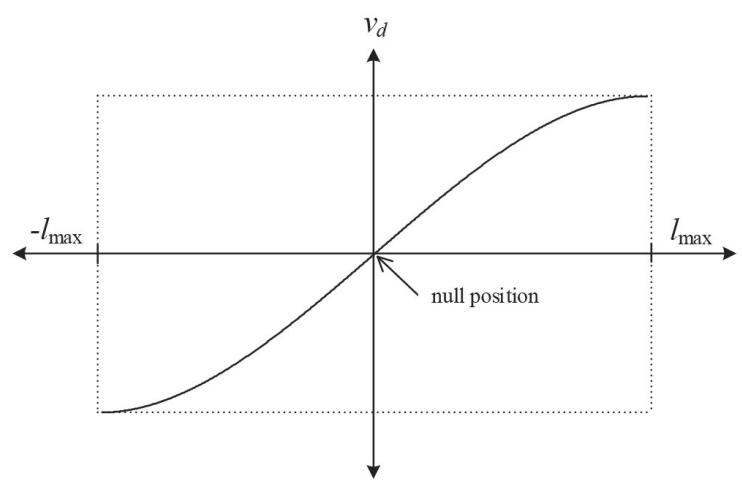

(b)

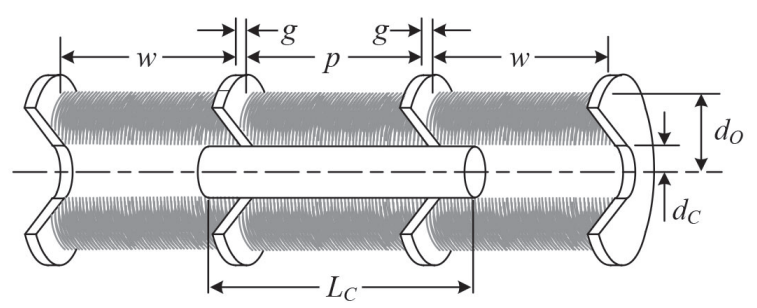

(c)

Fig. 1. (a) Principle, (b) output signal, and (c) structure of LVDT.

and $\omega_{e x}$ are the amplitude and frequency of the sinusoidal signal, respectively. If only the magnitude of the LVDT signal is considered, then the secondary winding signals $v_{S 1}$ and $v_{S 2}$ can be expressed as ${ }^{(12,22,23)}$

$$
v_{S 1}=\frac{2 \pi^{2} \omega_{e x} V_{e x} n_{P} n_{S}\left(2 l_{2}+p\right)}{10^{7} w L_{C} Z_{P} \ln \left(d_{O} / d_{C}\right)} l_{1}^{2},
$$

and

$$
v_{S 2}=\frac{2 \pi^{2} \omega_{e x} V_{e x} n_{P} n_{S}\left(2 l_{1}+p\right)}{10^{7} w L_{C} Z_{P} \ln \left(d_{O} / d_{C}\right)} l_{2}^{2},
$$

where $Z_{P}$ denotes the impedance of the primary winding, and $l_{1}$ and $l_{2}$ are the lengths of the core penetrating into the secondary windings $S_{1}$ and $S_{2}$, respectively. From the structure of the LVDT, the displacement signal can be obtained from the difference signal between two secondary winding signals, $v_{d}=\left(v_{S 1}-v_{S 2}\right)$, and can be stated as

$$
v_{d}=K_{1} l\left(1-K_{2} l^{2}\right),
$$


for $K_{1}=\frac{8 \pi^{2} \omega_{e x} V_{e x} n_{P} n_{S}\left(p+2 g+l_{0}\right) l_{0}}{10^{7} w L_{C} Z_{P} \ln \left(d_{O} / d_{C}\right)}$, and $K_{2}=\frac{1}{\left(p+2 g+l_{0}\right) l_{0}}$, where $l_{0}=\left(l_{1}+l_{2}\right) / 2$ is the average length of the core penetrating into the secondary windings $S_{1}$ and $S_{2}$, and $l=\left(l_{1}-l_{2}\right) / 2$ is the core position. Practically, the length $p$ is larger than the gap $g$ or $p \gg>g$. From Fig. 1(c), the length $L_{C}$ can be approximated as $L_{C}=3 p$; then, the difference signal $v_{d}$ of Eq. (2) can be given by $(4,12,24)$

$$
v_{d}=\frac{8 \pi^{2} \omega_{e x} V_{e x} n_{P} n_{S}}{10^{7} Z_{P} \ln \left(d_{O} / d_{C}\right)} \frac{2 p}{3 w}\left(l-\frac{l^{3}}{2 p^{2}}\right)=k_{t} l\left(1-k_{n} l^{2}\right),
$$

where $k_{t}$ and $k_{n}$ are the sensitivity and nonlinear coefficient of the LVDT, respectively. The difference signal $v_{d}$ of Eq. (3) exhibits only the amplitude of the AMSC from the LVDT output signal versus the excitation signal. To extract the displacement signal from the LVDT output signal, the synchronous demodulator is required to generate the envelope of the difference signal $v_{d}$. Note that the response of the demodulated signal is nonlinear along the entire core position, as shown in Fig. 1(b). The linear range of the LVDT transfer characteristic lies in a narrow range close to the zero crossing of the LVDT response curve. The LVDT signal $v_{d}$ can be approximated as linear for the moving core varying in the narrow range close to the zero crossing of the LVDT transfer characteristic curve in Fig. 1(b). Thus, the difference signal $v_{d}$ can be expressed as being in linear proportion to the core position $l$ as

$$
v_{d}=k_{t} l
$$

From Eqs. (2) and (3), the linear range defined by $l_{L}$ of Eq. (4) is limited by the nonlinear coefficient $k_{n}$. Practically, the nonlinear coefficient $k_{n}$ is described in terms of the acceptable error $\varepsilon_{L}$ of the LVDT output signal. Therefore, the linear range $l_{L}$ can be expressed as ${ }^{(23)}$

$$
l_{L}= \pm \sqrt{\frac{\varepsilon_{L}}{k_{n}}} .
$$

From the specifications of the LVDT used in this work, the acceptable error $\varepsilon_{L}$ is equal to $0.5 \%$ and $k_{n}=1.106 \times 10^{-3} \mathrm{~V} / \mathrm{mm}^{2}$; then, the linear range $l_{L}$ is about $\pm 2.13 \mathrm{~mm}$.

\section{Proposed Technique}

The nonlinear term in parentheses in Eq. (3) causes the narrow linear operating range of the LVDT. This nonlinear term can be compensated using the inverse property $N(l)$ to extend the linear operating range as

$$
N(l)=\frac{1}{\left(1-\alpha v_{d}^{2}\right)},
$$


where $\alpha$ is the weighting coefficient used to minimize the error of the displacement signal. Practically, the magnitude of the term $k_{n} l^{2}$ in Eq. (3) is $\left|k_{n} l^{2}\right|<1$. This is because the nonlinear coefficient $k_{n}$ of a commercial LVDT is very small and unaffected when the displacement $l$ approaches zero. Consequently, the weighting coefficient $\alpha$ in Eq. (6) is $\alpha<1$. If the maximum value of the displacement signal $v_{d}$ is assigned for the condition of $\left|\alpha v_{d}^{2}\right|<1$, then Eq. (6) can be expanded using the binomial series as ${ }^{(24)}$

$$
N(l)=1+\alpha v_{d}^{2}+\left(\alpha v_{d}^{2}\right)^{2}+\left(\alpha v_{d}^{2}\right)^{3}+\cdots
$$

From Eq. (7), the higher order terms can be neglected when $\left|\alpha v_{d}^{2}\right|<1$. Then, the nonlinear term $N(l)$ can be approximated as

$$
N(l)=1+\alpha v_{d}^{2} .
$$

From Eq. (3), the nonlinear term $\left(1-k_{n} l^{2}\right)$ can be compensated by the term $N(l)$ of Eq. (7). The block diagram for the linearization of the LVDT behavior is shown in Fig. 2(a). From Fig. 2(a), the output signal $v_{o l}$ versus the signal $v_{d}$ can be stated as

$$
v_{o l}=v_{d}+\alpha v_{d}^{3}=v_{d}\left(1+\alpha v_{d}^{2}\right) .
$$

From Eq. (9), if the displacement signal $v_{d}$ is applied, the output signal $v_{o l}$ can be given by

$$
v_{o l}=k_{t} l+\left[\left(\alpha k_{t}^{3}-k_{t} k_{n}\right) l^{3}-3 \alpha k_{t}^{3} k_{n} l^{5}+3 \alpha k_{t}^{3} k_{n}^{2} l^{7}-\alpha k_{t}^{3} k_{n}^{3} l^{9}\right] .
$$

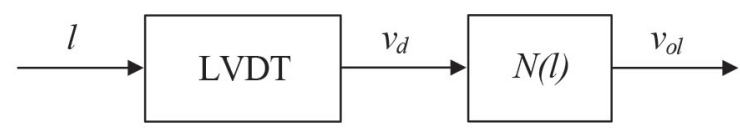

(a)

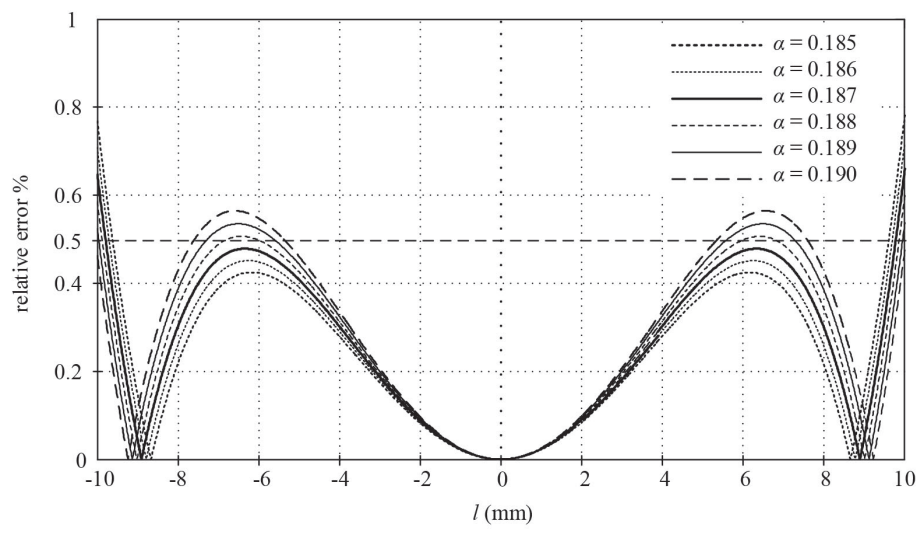

(b)

Fig. 2. (a) Principle of LVDT. (b) Plot of Eq. (12). 
If an appropriate value of the weighting coefficient $\alpha$ is chosen, then the output signal $v_{o l}$ in Eq. (10) can be approximated as

$$
v_{o l}=k_{t} l
$$

The relative error of the output signal $v_{o l}$, defined by $\varepsilon_{o l}$, can be expressed as

$$
\varepsilon_{o l}=\left[\left(\alpha k_{t}^{2}-k_{n}\right) l^{2}-3 \alpha k_{t}^{2} k_{n} l^{4}+3 \alpha k_{t}^{2} k_{n}^{2} l^{6}-\alpha k_{t}^{2} k_{n}^{3} l^{8}\right] \times 100 \%
$$

The plot of Eq. (12) is shown in Fig. 2(b) and used to determine the coefficient $\alpha$. From Fig. 2(b), if the relative error $\varepsilon_{o l}$ of about $0.5 \%$ is assigned to equal the specification of the LVDT used in this work with the linear stroke range of about $\pm 1.9 \mathrm{~mm}$, then the weighting coefficient $\alpha=0.187$ is observed at the core position $l= \pm 9.8 \mathrm{~mm}$. Note that the linear stroke range can be extended from \pm 1.9 to $\pm 9.8 \mathrm{~mm}$. The circuit diagram containing the property of Eq. (9) can be simply implemented using the analog multipliers and summing amplifier, as shown in Fig. 3(a). Note that the commercial analog multiplier provides an attenuation factor $\eta$. The summing amplifier comprises an operational amplifier (opamp), resistors $R_{1}-R_{3}$, and variable resistor $R_{n}$, as shown inside the dashed-line block in Fig. 3(a). Therefore, the output signal $v_{o l}$ in Fig. 3(a) can be given by

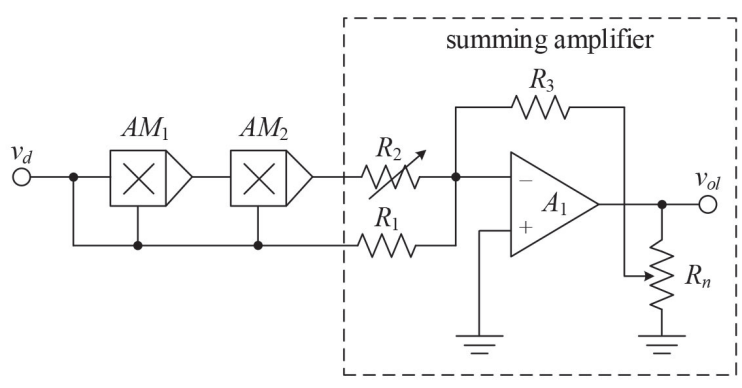

(a)

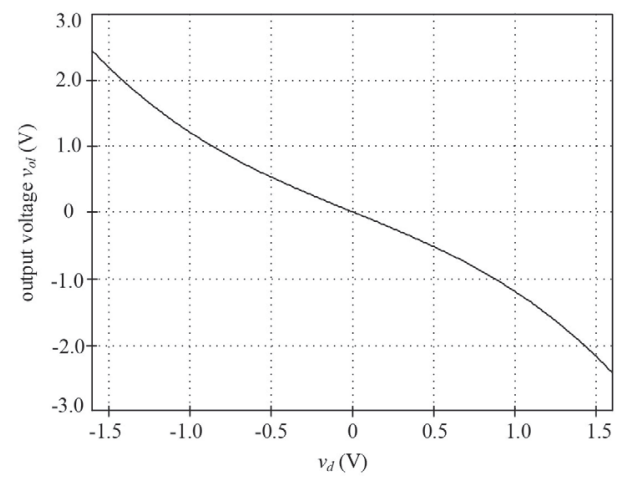

(b)

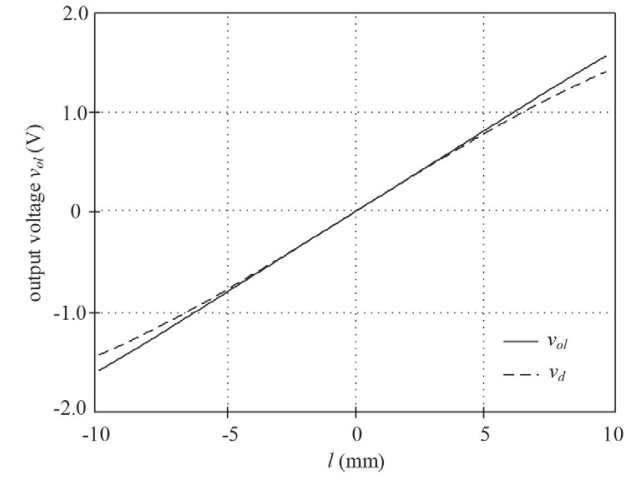

(c)

Fig. 3. (a) Proposed circuit, (b) simulation result of reverse transfer characteristic, and (c) linearization of LVDT. 


$$
v_{o l}=-\frac{R_{3}}{\beta R_{1}}\left(1+\frac{R_{1}}{R_{2}} \eta v_{d}^{2}\right) v_{d},
$$

where $\beta$ is the amplification factor used to span the output signal $v_{o l}$ to the appropriate value and to compensate nonideal values between resistors $R_{3}$ and $R_{1}$. Note that the output signal $v_{o l}$ of the scheme in Fig. 3(a) is $180^{\circ}$ out of phase. The parameters of the LVDT used in this work are measured from the experimental implementation as $k_{t}=84.817 \mathrm{mV} / \mathrm{mm} / \mathrm{V}, k_{n}=$ $1.106 \times 10^{-3} \mathrm{~mm}^{-2}$, and $l_{L}= \pm 1.9 \mathrm{~mm}$ for the relative error of $0.5 \%$. The parameter $k_{n}$ can be calculated from a maximum value of the transfer characteristic curve of the LVDT and Eq. (3) as

$$
k_{n}=\frac{k_{t} l_{m}-v_{d m}}{k_{t} l_{m}^{3}},
$$

where $v_{d m}$ and $l_{m}$ are the maximum value of the LVDT transfer characteristic curve and the position along the length of the moving core at the maximum voltage $v_{d m}$, respectively. Simulation results of the LVDT reverse transfer characteristic in Fig. 3(b) and the linearization of the LVDT behavior using the proposed technique for an excitation signal with the peak amplitude of $1 \mathrm{~V}$ in Fig. 3(c) were obtained using the PSPICE analog simulation program. It can be seen that the linear operating range of the LVDT used in this study can be extended more than fivefold.

\section{Performance of Proposed Scheme}

Deviation from the ideal performance of the proposed scheme is caused by the nonideal characteristics of the analog multipliers and the tolerance of the resistors used in the summing amplifier. From Fig. 3(a), the output signal $v_{o l}$ caused by the errors of the analog multipliers and the nonideal factor between the resistors $R_{1}$ and $R_{2}$, defined as $\Delta_{12}$, can be expressed as

$$
v_{o l}=-\frac{R_{3}}{\beta R_{1}} v_{d}\left(1+\frac{R_{1}}{R_{2}} \eta v_{d}^{2}\right)-\left(\Delta_{12}+\varepsilon_{m 12}\right) \frac{R_{3}}{\beta R_{2}} \eta v_{d}^{3},
$$

where $\varepsilon_{m 12}$ denotes the error of the analog multipliers. From Eq. (15), the amplification factor $\beta$ can be varied to assign the term $\left(R_{3} / \beta R_{1}\right)=K_{L}$, where $K_{L}$ is the gain of the proposed scheme. Therefore, Eq. (15) can be rewritten as

$$
v_{o l}=-K_{L} v_{d}\left(1+\frac{R_{1}}{R_{2}} \eta v_{d}^{2}\right)-\left(\Delta_{12}+\varepsilon_{m 12}\right) K_{L} \frac{R_{1}}{R_{2}} \eta v_{d}^{3} .
$$

From Eq. (16), the second term on the right-hand side, that is, the error term, can be minimized by replacing the resistor $R_{2}$ with the variable resistor. If the resistance $R_{2}$ is varied 
to approach the expected value, then the relative error of the output signal $v_{o l}$, defined as $\varepsilon_{o c}$, can be expressed as

$$
\varepsilon_{o c}=\frac{\eta R_{1}\left(\Delta_{12}+\varepsilon_{m 12}\right) v_{d}^{2}}{R_{2}\left(1+\frac{R_{1}}{R_{2}} \eta v_{d}^{2}\right)} \times 100 \%
$$

If the resistances $R_{1}$ and $R_{2}$ are chosen to satisfy the nonideal factor $\Delta_{12}$ of about $0.2 \%$, error $\varepsilon_{m 12}=0.4 \%,\left(\eta R_{1} / R_{2}\right)=0.187$, and the peak amplitude of the signal $v_{d}=762.97 \mathrm{mV}$ corresponding to the core displacement $l=9.8 \mathrm{~mm}$, then the relative error $\varepsilon_{o c}$ of about $0.06 \%$ is calculated. Note that the relative error $\varepsilon_{o c}$ is very small. Therefore, the inaccuracy of the proposed technique corresponding to the relative error $\varepsilon_{o c}$ can be neglected. From Eqs. (12) and (17), it can be seen that the major factor contributing to the inaccuracy of the proposed technique is the relative error $\varepsilon_{o l}$.

\section{Experimental Results}

The proposed scheme in Fig. 3(a) was constructed using commercially available devices to demonstrate its performance, as shown in Fig. 4(a). The active devices used in this study were the analog multiplier AD633 and opamp LF351. The analog multiplier AD633 provides an attenuation factor of about 0.1 . Therefore, $\eta=0.01$ was obtained. The resistors $R_{1}=R_{3}=20 \mathrm{k} \Omega$ with $0.2 \%$ tolerance were chosen. From Eq. (12) and Fig. 2(b), $\eta R_{1} / R_{2}$ was equal to 0.187 . Thus, the resistance $R_{2}$ was calculated as $10.7 \mathrm{k} \Omega$. The resistor $R_{2}$ was replaced by the variable resistor to tune the resistance to be close to the calculated value. The variable resistor $R_{n}$ of $1 \mathrm{k} \Omega$ was chosen. The power supply was set as $\pm 9 \mathrm{~V}$. The nonlinearity error of the term $v_{d}{ }^{2}$ was measured to be about $0.4 \%$ or $\varepsilon_{m 12}=4 \times 10^{-3}$. The commercially available LVDT used in this study provides the following specifications: linear stroke range of $\pm 2 \mathrm{~mm}$, maximum relative error of $0.5 \%$, and sensitivity $k_{t}=94.5 \mathrm{mV} / \mathrm{mm} / \mathrm{V}$. The excitation signal applied to

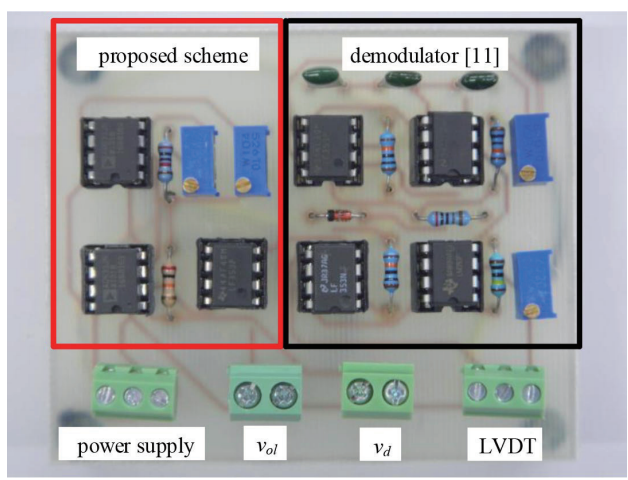

(a)

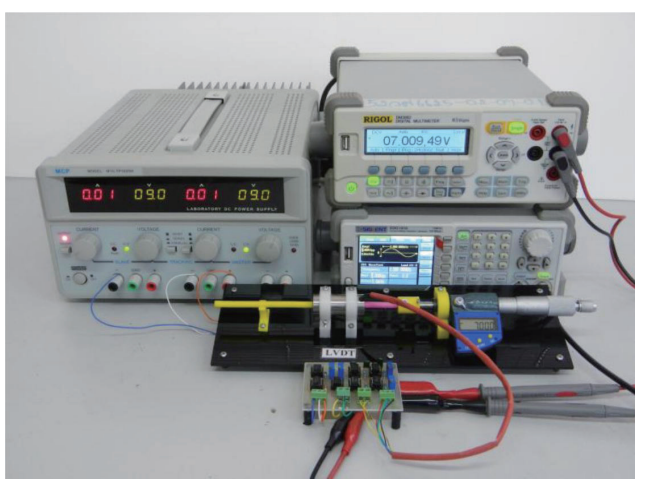

(b)

Fig. 4. (Color online) (a) Proposed circuit. (b) Experimental setup. 
the LVDT is set as a $2.5 \mathrm{kHz}$ sinusoidal wave with a $2 \mathrm{~V}$ peak-to-peak amplitude. In this experiment, the output signal of the LVDT is demodulated using the technique proposed in the literature $^{(13)}$. The experimental setup is shown in Fig. 4(b). The practical behavior of the LVDT is measured and shown in Fig. 5 to extract the parameters. The parameters of the LVDT can be determined as follows. The sensitivity of the LVDT is determined to be $84.817 \mathrm{mV} / \mathrm{mm} / \mathrm{V}$.

The maximum amplitude of the LVDT signal $v_{d m}$ is $971.55 \mathrm{mV}$ at the maximum stroke or $l_{m}= \pm 16 \mathrm{~mm}$, and the linear range of the LVDT is about $\pm 1.9 \mathrm{~mm}$ for the relative error of $0.5 \%$. The nonlinear coefficient $k_{n}$ can be calculated using Eq. (14) as $1.106 \times 10^{-3} \mathrm{~V} / \mathrm{mm}^{2}$. The LVDT inverse transfer characteristic of the proposed scheme is shown in Fig. 6(a), where the difference signal $v_{d}$ is assigned as $\left(v_{s 2}-v_{s 1}\right)$ or $-v_{d}$ for the inverse operation of the signal $v_{o l}$. The demodulated LVDT signal is synthesized using the LabVIEW program and analog input/ output board from National Instruments (NI-USB-6009) to demonstrate the performance of the proposed technique, where the LVDT parameters are set to the same parameters as the practical LVDT used in this study. The synthesized signal is applied to the scheme in Fig. 3(a) for the peak amplitude of $\pm 971.55 \mathrm{mV}$ corresponding to the core position of $\pm 16 \mathrm{~mm}$. The measured

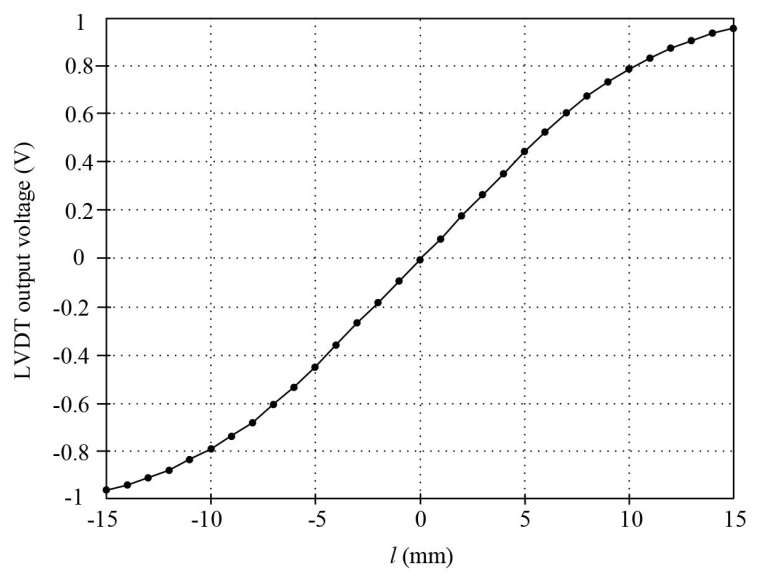

Fig. 5. Practical behavior of LVDT.

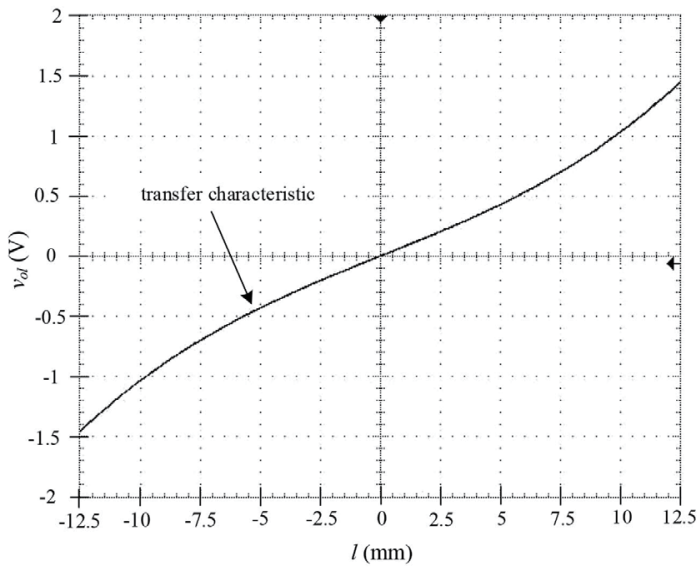

(a)

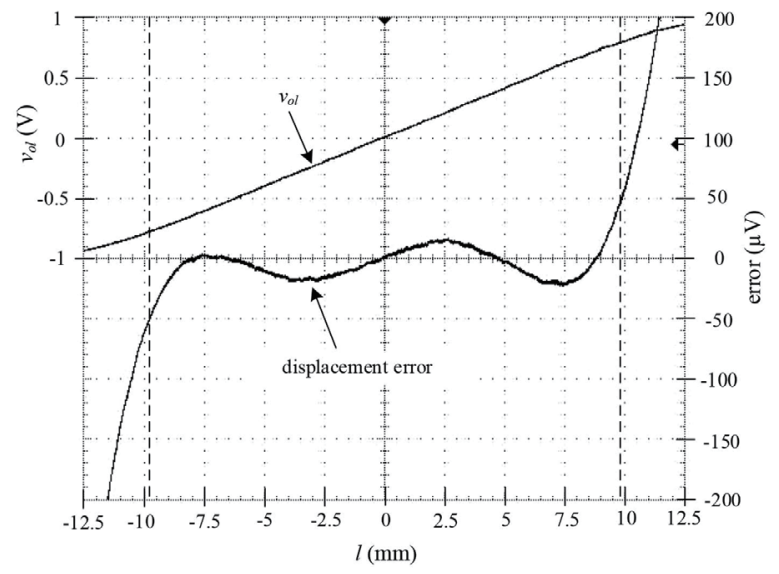

(b)

Fig. 6. (a) Transfer characteristic of the proposed scheme. (b) Measured result of the proposed scheme. 


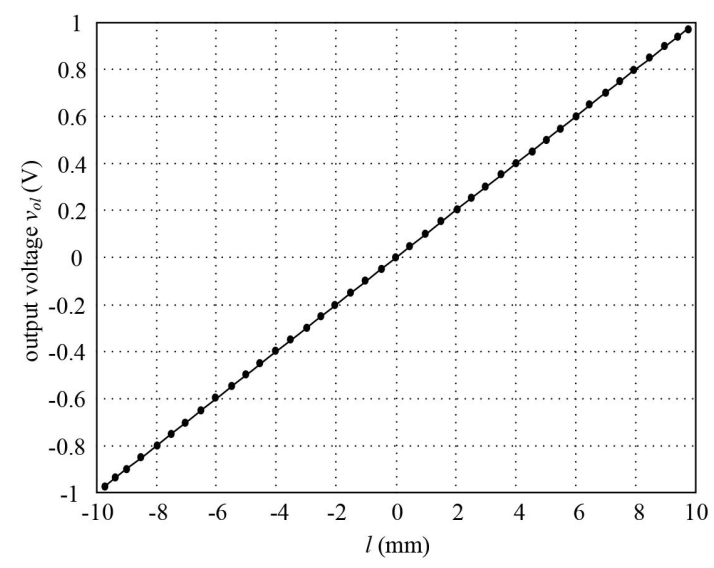

(a)

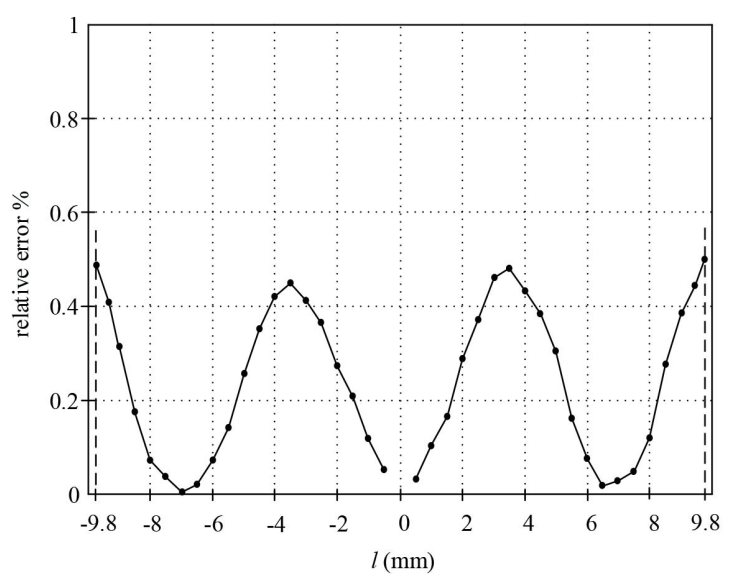

(b)

Fig. 7. (a) Measured result of the output signal. (b) Relative error.

result confirmed the proposed technique performance, as shown in Fig. 6(b). It can be seen that the proposed technique can extend the linear operating range from \pm 1.9 to $\pm 9.8 \mathrm{~mm}$. Figure 7 (a) shows the plot of the average voltage of the output signal $v_{o l}$ from the circuit in Fig. 3(a) of 5 repeated measurements for each core position from -9.8 to $9.8 \mathrm{~mm}$. The sensitivity of the proposed scheme is set to $100 \mathrm{mV} / \mathrm{mm} / \mathrm{V}$ by adjusting the variable resistor $R_{n}$ to vary the amplification factor $\beta$. The relative error $\varepsilon_{r}$ of the measured signal $v_{o l}$ is shown in Fig. 7(b). The maximum relative error $\varepsilon_{r}$ of the measured result in Fig. 7(b) corresponding to the absolute error $\varepsilon_{a m}$ of about $46 \mu \mathrm{m}$ is observed, where the maximum relative error $\varepsilon_{r}$ occurs at the core position of $\pm 9.8 \mathrm{~mm}$. The full-scale error of the proposed technique, defined as $\varepsilon_{f s}$, can be stated as

$$
\varepsilon_{f s}=\frac{\max \left(\varepsilon_{\text {am }}\right)}{\mid \max (\text { stroke range }) \mid} \times 100 \% .
$$

If the maximum absolute error $\varepsilon_{a m}$ is equal to $46 \mu \mathrm{m}$, the full-scale error $\varepsilon_{f s}$ is calculated to be about $0.23 \%$.

\section{Conclusions}

The inverse transfer characteristic of an LVDT based on a binomial series was proposed in this paper. A simple circuit configuration comprising analog multipliers and an opamp was adopted for synthesizing the LVDT inverse transfer characteristic without a specific device. The linear operating range of the LVDT was extended more than fivefold from the LVDT specification used in this study, that is, from \pm 1.9 to $\pm 9.8 \mathrm{~mm}$. The performance of the proposed circuit was confirmed by experimental implementation. The proposed circuit can be used as a signal conditioner to measure a large displacement using a small-structure LVDT with very good performance. The proposed technique exhibits a simple configuration that is suitable for measurement systems used in small areas such as a miniature robot and an embedded system. 


\section{Acknowledgments}

This work was supported by the King Mongkut's Institute of Technology Ladkrabang (KMITL), Thailand, under Grant KREF115701.

\section{References}

1 R. Pallas-Areny and J. G. Webster: Sensor and Signal Condition (John Wiley \& Sons, New York, 2001) p. 229.

2 K. Ara: IEEE Trans. Instrum. Meas. 21 (1972) 249. https://doi.org/10.1109/TIM.1972.4314011

3 S. C. Saxena and S. B. L. Seksena: IEEE Trans. Inst. Meas. 38 (1989) 748. https://doi.org/10.1109/19.32186

4 H. K. P. Neubert: Instrument Transducers - An Introduction to Their Performance and Design (Oxford, New York, 1975) p. 204.

5 S. Cetinkunt: Mechatronics (John Wiley \& Sons, New York, 2006) p. 227.

6 M. Felix, A. Lizarraga, A. Islas, and A. Gonzales: 36th Annual Conf. IEEE Industrial Electronics Society (2010) 1772.

7 R. M. Ford, R. S. Weissbach, and D. R. Loker: 17th IEEE Instrumentation and Measurement Technology Conf. (IMTC/2000) 1448.

8 S. Lee and W. Kim: IEEE Trans. Control Syst. Tech. 18 (2010) 859. https://doi.org/10.1109/TCST.2009.2030413

9 G. Chen, B. Zhang, P. Liu, and H. Ding: IEEE Sens. J. 15 (2015) 2248. https://doi.org/10.1109/ JSEN.2014.2364610

10 W. Petchmaneelumka, A. Rerkratn, A. Luangpol, and V. Riewruja: J. Circ. Syst. Comp. 27 (2018) 1850182. https://doi.org/10.1142/S0218126618501827

11 W. Petchmaneelumka, P. Mano, and V. Riewruja: Sens. Mater. 30 (2018) 2171. https://doi.org/10.18494/ SAM.2018.1816

12 P. Veeraian, U. Gandhi, and U. Mangalanathan: Int. J. Electron. Commun. 79 (2017) 141. https://doi. org/10.1016/j.aeue.2017.05.037

13 W. Petchmaneelumka, K. Songsuwankit, and V. Riewruja: I.R.E.E. 11 (2016) 340. https://doi.org/10.15866/ iree.v11i3.8906

14 W. Petchmaneelumka, P. Mano, K. Songsuwankit, and V. Riewruja: Int. J. Electron. 105 (2018) 1520. https:// doi.org/10.1080/00207217.2018.1461250

15 V. Riewruja and T. Kamsri: IET Circ. Device Syst. 3 (2009) 57. https://doi.org/10.1049/iet-cds.2008.0140

16 V. Riewruja and A. Rerkratn: Int. J. Electron. 98 (2011) 459.

17 V. Riewruja and A. Rerkratn: Indian J. Pure Appl. Phys. 48 (2010) 67.

18 A. Drumea, A. Vasile, M. Comes, and M. Blejan: 1st Electronics System Integration Technology Conference (2006) 629.

19 K. Banerjee, B. Dam, and K. Majumdar: IEEE Int. Symp. Industrial Electronics (2013) 1. https://doi. org/10.1109/ISIE.2013.6563715

20 R. Casanella, O. Casas, and R. Pallas-Areny: Meas. Sci. Technol. 16 (2005) 1637. https://doi.org/10.1088/09570233/16/8/014.

21 R. Casanella, O. Casas, M. Ferrari, V. Ferrari, and R. Pallas-Areny: IEEE Trans. Instrum. Meas. 56 (2007) 1219. https://doi.org/10.1109/TIM.2007.899919.

22 S. R. Mishra, G. Panda, and D. P. Das: IEEE Trans. Instrum. Meas. 59 (2010) 947. https://doi.org/10.1109/ TIM.2009.2031385.

23 W. Petchmaneelumka, W. Koodtalang, and V. Riewruja : IEEE Sensors J. 19 (2019) 5045. https://doi. org/10.1109/JSEN.2019.2902879

24 M. R. Spiegel: Mathematical Handbook (Scaum's Outline Series, Singapore, 1968) p. 110. 